\title{
THE EFFECT AMOUNT NOMINAL STEAM FLOW ON THE GAS BURNER DESIGN ELEMENTS FOR STEAM GENERATOR
}

\author{
BAYDAA FAREED RAJAB \& WESAM ADEL ABDULAMEER \\ ${ }^{I}$ Mechanical Engineering, Mechanical and Mechatronics Engineering, University Politehnica of Bucharest, \\ Romania. Ministry of Electricity, Iraq, Babil \\ ${ }^{2}$ Material Engineering, Material Engineering and Science, University Politehnica of Bucharest, Romania. \\ Ministry of Electricity, Iraq, Babil
}

\begin{abstract}
To help designers understand Important and influencing elements for choosing the suitable gas burner directly from the charts without resorting to complex mathematical operations and When studying many variables that affect the work of the gas burner of steam boilers in a set of different operating conditions and after selecting a burner that relies on natural gas as fuel, the results of the study and the mathematical processes are presented about the effect of the design of openings and the dimensions of injection tube and dependence on the amount of nominal steam flow to be produce.

KEYWORDS: Design Burner, Steam Generator, Nominal Steam Flow \& Natural Gas
\end{abstract}

Received: Jun 05, 2020; Accepted: Jun 25, 2020; Published: Oct 05, 2020; Paper Id.: IJMPERDJUN20201475

\section{INTRODUCTION}

Burner an important part of the steam generator, its main function is to mix fuel and air in specific proportions to produce heat energy in a boiler furnace, The energy efficiency of a fuel burner depends on the type of fuel burned in the boiler because each type of fuel has different combustion properties. Gaseous fuel is usually the easiest to burn and control within a boiler. Liquid fuel is easy to handle and burn, while solid fuel is the most difficult [10]. Natural gas burns cleanly because it mixes with air easily and the products of combustion are water vapor and carbon dioxide, with small amounts of sulfur and nitrogen oxides. In general, complete combustion of natural gas can be achieved easily, and if the combustion equipment is well-maintained, in addition to making precise designs for the burner to obtain the largest amount of heat resulting from the combustion process and avoid heat loss and increase efficiency.[4,5]

\section{MATHEMATICAL CALCULATION}

Burner an important part of the steam generator, its main function is to mix fuel and air in specific proportions to produce heat energy in a boiler furnace, The energy efficiency of a fuel burner depends on the type of fuel burned in the boiler because each type of fuel has different combustion properties. Gaseous fuel is usually the easiest to burn and control within a boiler. Liquid fuel is easy to natural gas can be achieved easily, and if the combustion equipment is well-maintained, in addition to making precise designs for the handle and burn, while solid fuel is the most difficult[10]. Natural gas burns cleanly because it mixes with air easily and the products of combustion are $\mathrm{H}_{2} \mathrm{O}$ vapor and $\mathrm{Cr}$ dioxide, with small amounts of $\mathrm{SO}_{2}$ and $\mathrm{NO}_{\mathrm{X}}$. In general, complete combustion of burner to obtain the largest amount of heat resulting from the combustion process and avoid heat loss and increase efficiency.[4,5] 


\section{Equations and Data For Use}

These values were chosen to design a steam boiler generator that produces a nominal steam flow [ Dn ] between (10 - 50 ) $[\mathrm{T} / \mathrm{h}]$.

Table 1: Nominal Steam Flow

\begin{tabular}{|l|l|l|l|l|l|}
\hline 10 & 18 & 26 & 34 & 42 & 50 \\
\hline 12 & 20 & 28 & 36 & 44 & \\
\hline 14 & 22 & 30 & 38 & 46 & \\
\hline 16 & 24 & 32 & 40 & 48 & \\
\hline
\end{tabular}

Table 2: Operational Conditions

\begin{tabular}{|l|l|l|l|l|l|}
\hline Sy. & \multicolumn{1}{|c|}{ Quantity } & \multicolumn{1}{c|}{ Value } & \multicolumn{1}{c|}{ Sy. } & \multicolumn{1}{c|}{ Quantity } & \multicolumn{1}{c|}{ Value } \\
\hline tn & nominal temperature of the steam & $180\left[{ }^{\circ} \mathrm{C}\right]$ & $\mathrm{d}$ & humidity of furnace gas & $18[\mathrm{~g} / \mathrm{m} 3 \mathrm{~N}]$ \\
\hline $\mathrm{pn}$ & nominal pressure of the steam & $10[\mathrm{bar}]$ & $\lambda$ & coefficient for exhaust air & 1.1 const. \\
\hline $\mathrm{t}^{\prime} \mathrm{e}$ & temperature of supply water & $60\left[{ }^{\circ} \mathrm{C}\right]$ & $\mathrm{pref}$ & reference pressure & $760[\mathrm{mmHg}]$ \\
\hline tev & temperature of the flue exhaust gas & $220\left[{ }^{\circ} \mathrm{C}\right]$ & tref & reference temperature & $0\left[{ }^{\circ} \mathrm{C}\right]$ \\
\hline tp & temperature of the preheated & $220\left[{ }^{\circ} \mathrm{C}\right]$ & $\mathrm{p}$ & atmospheric pressure & $1.013[\mathrm{bar}]$ \\
\hline $\mathrm{pe}^{\prime}$ & pressure of supply water & $11[\mathrm{bar}]$ & $\mathrm{pr}$ & pressure in a manifold & $4000[\mathrm{mmH} 2 \mathrm{O}]$ \\
\hline to & temperature of the gas & $25\left[{ }^{\circ} \mathrm{C}\right]$ & $\rho \mathrm{\rho}$ & air density & $1.293[\mathrm{~kg} / \mathrm{m} 3 \mathrm{~N}]$ \\
\hline $\mathrm{X}$ & air humidity & $10[\mathrm{~g} / \mathrm{kg}]$ & $\mathrm{\rho g}$ & density of natural gas & $0.72[\mathrm{~kg} / \mathrm{m} 3 \mathrm{~N}]$ \\
\hline
\end{tabular}

P00ref, tref In ideal conditions, Nominal temperature and pressure:- the pressure and temperature of the superheated steam from a boiler, $\mathrm{p}=1.013$ [bar] $\rightarrow 101300[\mathrm{pa}], \mathrm{pr}=4000\left[\mathrm{~mm} \mathrm{H}_{2} \mathrm{O}\right] \rightarrow 39240[\mathrm{pa}]$

Table 3: Analysis of natural gas

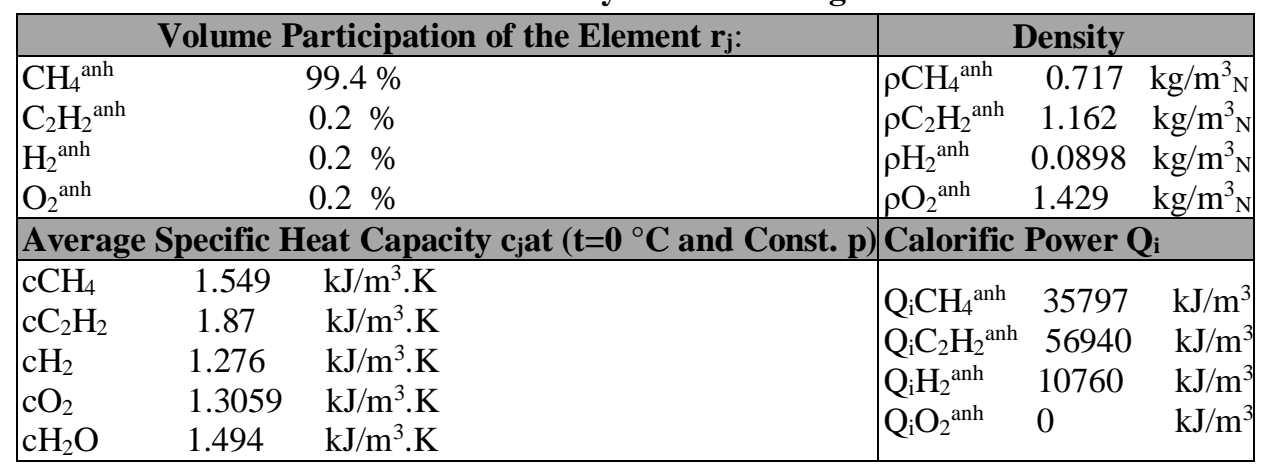

- Calorific power Qi:- The amount of energy produced by the complete combustion of a material or fuel.

- Average specific heat capacity $\mathbf{c}_{\mathbf{j}}$ :- the amount of heat required to raise the unit temperature of a unit mass of a body

\section{Gas Pipe Diameter}

The diameter of the gas conductor pipe to the burner Di[m] increases with increasing amount of vapor to be produced, and we take the value $\mathrm{Di}$ at $\mathrm{Dn}(12,14,16[\mathrm{~T} / \mathrm{h}])$ equal to $\mathrm{Di}$ at $(20[\mathrm{~T} / \mathrm{h}]$ because they are values very near etc.

Di at Dn $(10[\mathrm{t} / \mathrm{h}])=0.2 \mathrm{~m}$ const.

$\operatorname{Dn}(20[\mathrm{~T} / \mathrm{h}])=\operatorname{Di}$ at $(10[\mathrm{~T} / \mathrm{h}]) \cdot \sqrt{ } 2=0.282842712[\mathrm{~m}]$

$\operatorname{Dn}(30[\mathrm{~T} / \mathrm{h}])=\operatorname{Di}$ at $(20[\mathrm{~T} / \mathrm{h}]) \cdot \sqrt{ } 2=0.4[\mathrm{~m}]$ 


$$
\begin{aligned}
& \operatorname{Dn}(40[\mathrm{~T} / \mathrm{h}])=\operatorname{Di} \text { at }(30[\mathrm{~T} / \mathrm{h}]) \cdot \sqrt{ } 2=0.565685425[\mathrm{~m}] \\
& \operatorname{Dn}(50[\mathrm{~T} / \mathrm{h}])=\operatorname{Di} \text { at }(40[\mathrm{~T} / \mathrm{h}]) \cdot \sqrt{ } 2=0.642[\mathrm{~m}]
\end{aligned}
$$

\section{Gas Heating Value Calculator [1]}

$$
\begin{aligned}
& f 1-2=\frac{100}{(100+0.1242 \cdot d)} \\
& Q i^{\text {anh }}=0.01 \Sigma r j \cdot Q i_{j} \\
& Q i^{u m}=f 1-2 \cdot\left(Q i^{\text {anh }}-2.51 \cdot d\right)
\end{aligned}
$$

Where $(f 1-2)$ factor to pass from the dry gas to the wet, $\left(\mathrm{Qi}^{\mathrm{anh}}\right)$ calorific power for dry gas $\left[\mathrm{kJ} / \mathrm{m}^{3} \mathrm{~N}\right],\left(\mathrm{Qi}^{\mathrm{um}}\right)$ calorific power for moist gas $\left[\mathrm{kJ} / \mathrm{m}^{3} \mathrm{~N}\right]$.

\section{Volumes of Air and Gases Combustion [1,10]}

$$
\begin{aligned}
& V o_{\min }=0.01 \cdot\left[0.5 \cdot C o^{a n h}+0.5 \cdot H_{2}^{a n h}+1.5 \cdot H_{2} S^{a n h}+\left(\Sigma\left(m+\frac{n}{4}\right) \cdot C_{m} H_{n}^{a n h}\right)-O_{2}^{a n h}\right] \\
& V^{0} a=100 \cdot \frac{V o_{\min }}{21} \\
& V_{\text {aum }}^{0}=(1+0.00161 \cdot x) \cdot V^{0} a \\
& V_{C O_{2}}=0,01 \cdot\left(C O_{2}^{a n h}+C o^{a n h}+\Sigma m \cdot C_{m} H_{n}^{a n h}\right) \\
& V S O_{2}=0,01 \cdot\left(S_{2}^{a n h}+H_{2} S^{a n h}\right) \\
& V_{\mathrm{RO} 2}=\mathrm{VCO}_{2}+\mathrm{VSO}_{2} \\
& V_{N 2}^{0}=0,79 \cdot V^{0} a+0,01 \cdot N_{2}^{a n h} \\
& V_{g u}^{0}=V_{R O 2}+V_{N 2}^{0} \\
& V_{H 2 O}^{0}=0.01 \cdot\left(H_{2}^{a n h}+H_{2} S^{a n h}+0.5 \cdot \Sigma n \cdot C_{m} H_{n}^{a n h}+0.01242 \cdot d\right)+0,00161 \cdot x \cdot V^{0} a \\
& V_{g}^{0}=V_{g u}^{0}+V_{H 2 O}^{0} \\
& V a=\lambda \cdot V^{0} a \\
& V_{\text {aum }}=\lambda \cdot V_{\text {aum }}^{0} \\
& C c^{a n h}=0.01 \cdot \Sigma r_{j} \cdot c_{j} \\
& C c^{u m}=f 1-2 \cdot\left(C c^{a n h}+1.242 \cdot 10^{-3} \cdot d \cdot c H_{2} O\right)
\end{aligned}
$$

- $\quad$ Use Table 2 and 3 to find value for Eq. $(1,2, \ldots .17)$.

- (keep the above results in Table 4), This Table is important to find enthalpy theoretical of humid air, enthalpy of combustion gas at different temperature.

$\mathrm{V}^{0} \mathrm{a}, \mathrm{V}^{0}{ }_{\text {aum }}, \mathrm{V}_{\mathrm{min}}$ volume strictly necessary of dry air, moist air and oxygen $\left[\mathrm{m}^{3} \mathrm{~N} / \mathrm{m}^{3} \mathrm{~N}\right]$.

$\mathrm{Va}, \mathrm{V}_{\text {aum }}$ actual volume of dry air and moist air $\left[\mathrm{m}^{3}{ }_{\mathrm{N}} / \mathrm{m}^{3} \mathrm{~N}\right] . \mathrm{Vco}_{2}, \mathrm{VsO}_{2}$ amount of carbon and sulfur dioxide 
$\left[\mathrm{m}^{3}{ }_{\mathrm{N}} / \mathrm{m}^{3} \mathrm{~N}\right] . \mathrm{V}_{\mathrm{g}}^{0}, \mathrm{~V}_{\mathrm{gu}}^{0}, \mathrm{~V}^{0} \mathrm{H}_{2}, \mathrm{~V}^{0}{ }_{\mathrm{N} 2}, \mathrm{~V}_{\mathrm{RO} 2}$ theoretical volume of wet flue gas, dry flue gas, water vapor, nitrogen and gas Triatomic $\left[\mathrm{m}^{3}{ }_{\mathrm{N}} / \mathrm{m}^{3} \mathrm{~N}\right]$.

$\mathrm{Cc}^{\text {anh }}, \mathrm{Cc}{ }^{\text {um }}$ specific heat of anhydrous gas fuel and moist gas fuel $\left[\mathrm{KJ} / \mathrm{m}^{3} \cdot \mathrm{K}\right],\left(\mathrm{m}^{3} \mathrm{~N}\right)$ normal cubic meters.

Table 4: Flue Gas Enthalpy for Natural Gas

\begin{tabular}{|c|c|c|c|c|c|c|c|c|c|c|c|c|}
\hline $\mathbf{t}$ & $\begin{array}{l}\mathbf{V}_{\text {RO }} \\
{\left[\mathrm{m}^{2}\right.}\end{array}$ & $\begin{array}{l}=0.998 \\
\left.\mathrm{~N} / \mathrm{m}^{3} \mathrm{~N}\right]\end{array}$ & & $\begin{array}{l}=7.496 \\
\left.\mathrm{~N} / \mathrm{m}^{3} \mathrm{~N}\right]\end{array}$ & $\begin{array}{c}\mathbf{V}^{\mathbf{0}} \mathrm{H}_{2} \\
{[\mathrm{~m}} \\
\end{array}$ & $\begin{array}{l}0=2.145 \\
\left.N^{n} / \mathrm{m}^{3} \mathrm{~N}\right]\end{array}$ & $\begin{array}{c}3+5 \\
+7\end{array}$ & $\underset{\left[\mathbf{I}^{\mathbf{0}} \mathbf{g}\right.}{\mathbf{I} / \mathbf{m}^{3}}$ & $\begin{array}{r}\mathbf{V}^{\mathbf{o}} \\
{[\mathrm{m}} \\
\mathrm{m}\end{array}$ & $\begin{array}{l}=9.638 \\
\left.\mathrm{~N} / \mathrm{m}^{3} \mathrm{~N}\right]\end{array}$ & $\mathbf{I}_{\text {aum }}^{\mathbf{0}}$ & 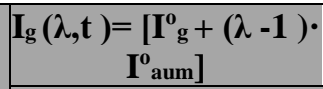 \\
\hline${ }^{\circ} \mathrm{C}$ & $\begin{array}{c}\mathrm{c}_{\mathrm{CO}} \\
2 \\
\end{array}$ & $\begin{array}{c}\mathbf{c}_{\mathrm{CO} 2} \cdot V_{\mathrm{R}} \\
02\end{array}$ & $c_{\mathrm{N} 2}$ & $\begin{array}{c}\mathbf{c}_{\mathrm{N} 2} \cdot \mathbf{V}^{\mathrm{o}} \mathrm{N} \\
2\end{array}$ & $\begin{array}{c}c \\
\mathrm{H} 2 \mathrm{O} \\
\end{array}$ & $\begin{array}{c}\mathrm{c}_{\mathrm{H} 2 \mathrm{O}} \cdot \mathrm{V}^{\mathrm{o}} \mathrm{H} \\
2 \mathrm{O}\end{array}$ & & $\mathrm{J} / \mathbf{m}^{2}$ & $\mathbf{c}_{\text {aum }}$ & $\begin{array}{c}c_{\text {aum }} \cdot \mathbf{V}^{\mathbf{0}} \\
\text { aum }\end{array}$ & $\begin{array}{c}{\left[\mathbf{k J} / \mathbf{m}^{3}\right.} \\
\mathrm{N}]\end{array}$ & $\lambda=1.1$ \\
\hline 1 & 2 & 3 & 4 & 5 & 6 & 7 & 8 & $\begin{array}{c}9=8 x \\
1\end{array}$ & 10 & 11 & $\begin{array}{c}12=11 \\
x 1\end{array}$ & 13 \\
\hline 0 & $\begin{array}{l}1.6 \\
00\end{array}$ & 1.597 & $\begin{array}{l}1.2 \\
94\end{array}$ & $\begin{array}{c}9.69 \\
9\end{array}$ & $\begin{array}{l}1.4 \\
94\end{array}$ & 3.204 & $\begin{array}{c}14.5 \\
00\end{array}$ & 0.000 & $\begin{array}{l}1.3 \\
18\end{array}$ & 12.703 & 0.000 & 0.000 \\
\hline 100 & $\begin{array}{l}1.7 \\
00\end{array}$ & 1.697 & $\begin{array}{l}1.2 \\
95\end{array}$ & $\begin{array}{c}9.70 \\
7\end{array}$ & $\begin{array}{l}1.5 \\
05\end{array}$ & 3.228 & $\begin{array}{c}14.6 \\
32\end{array}$ & $\begin{array}{c}1463.16 \\
6\end{array}$ & $\begin{array}{l}1.3 \\
24\end{array}$ & 12.761 & $\begin{array}{c}1276.12 \\
9\end{array}$ & 1590.779 \\
\hline 200 & $\begin{array}{l}1.7 \\
87\end{array}$ & 1.784 & $\begin{array}{l}1.2 \\
99\end{array}$ & $\begin{array}{c}9.73 \\
7\end{array}$ & $\begin{array}{l}1.5 \\
22\end{array}$ & 3.264 & $\begin{array}{c}14.7 \\
85\end{array}$ & $\begin{array}{l}2957.00 \\
5\end{array}$ & $\begin{array}{l}1.3 \\
31\end{array}$ & 12.829 & $\begin{array}{c}2565.75 \\
1\end{array}$ & 3213.580 \\
\hline 400 & $\begin{array}{l}1.9 \\
29\end{array}$ & 1.925 & $\begin{array}{l}1.3 \\
16\end{array}$ & $\begin{array}{c}9.86 \\
4\end{array}$ & $\begin{array}{l}1.5 \\
65\end{array}$ & 3.356 & $\begin{array}{c}15.1 \\
46\end{array}$ & $\begin{array}{c}6058.39 \\
7\end{array}$ & $\begin{array}{l}1.3 \\
54\end{array}$ & 13.050 & $\begin{array}{c}5220.17 \\
6\end{array}$ & 6580.415 \\
\hline 600 & $\begin{array}{l}2.0 \\
41\end{array}$ & 2.037 & $\begin{array}{l}1.3 \\
40\end{array}$ & $\begin{array}{c}10.0 \\
44\end{array}$ & $\begin{array}{l}1.6 \\
14\end{array}$ & 3.462 & $\begin{array}{c}15.5 \\
43\end{array}$ & $\begin{array}{c}9325.65 \\
4\end{array}$ & $\begin{array}{l}1.3 \\
82\end{array}$ & 13.320 & $\begin{array}{c}7992.19 \\
0\end{array}$ & 10124.873 \\
\hline 800 & $\begin{array}{l}2.1 \\
31 \\
\end{array}$ & 2.127 & $\begin{array}{l}1.3 \\
67 \\
\end{array}$ & $\begin{array}{c}10.2 \\
47 \\
\end{array}$ & $\begin{array}{l}1.6 \\
68 \\
\end{array}$ & 3.577 & $\begin{array}{c}15.9 \\
51\end{array}$ & $\begin{array}{c}12760.6 \\
21 \\
\end{array}$ & $\begin{array}{l}1.4 \\
11 \\
\end{array}$ & 13.600 & $\begin{array}{c}10879.8 \\
65 \\
\end{array}$ & 13848.607 \\
\hline $\begin{array}{c}100 \\
0\end{array}$ & $\begin{array}{l}2.2 \\
03\end{array}$ & 2.199 & $\begin{array}{l}1.3 \\
91\end{array}$ & $\begin{array}{c}10.4 \\
27\end{array}$ & $\begin{array}{l}1.7 \\
22\end{array}$ & 3.693 & $\begin{array}{c}16.3 \\
18 \\
\end{array}$ & $\begin{array}{c}16318.3 \\
44\end{array}$ & $\begin{array}{l}1.4 \\
37\end{array}$ & 13.850 & $\begin{array}{c}13850.4 \\
30\end{array}$ & 17703.387 \\
\hline $\begin{array}{c}120 \\
0\end{array}$ & $\begin{array}{l}2.2 \\
63\end{array}$ & 2.258 & $\begin{array}{l}1.4 \\
14\end{array}$ & $\begin{array}{c}10.5 \\
99\end{array}$ & $\begin{array}{l}1.7 \\
76\end{array}$ & 3.809 & $\begin{array}{c}16.6 \\
66\end{array}$ & $\begin{array}{c}19999.7 \\
29\end{array}$ & $\begin{array}{l}1.4 \\
61\end{array}$ & 14.082 & $\begin{array}{c}16898.1 \\
03\end{array}$ & 21689.539 \\
\hline $\begin{array}{c}140 \\
0\end{array}$ & $\begin{array}{l}2.3 \\
13\end{array}$ & 2.308 & $\begin{array}{l}1.4 \\
34\end{array}$ & $\begin{array}{c}10.7 \\
49\end{array}$ & $\begin{array}{l}1.8 \\
28\end{array}$ & 3.921 & $\begin{array}{c}16.9 \\
78\end{array}$ & $\begin{array}{c}23768.8 \\
93\end{array}$ & $\begin{array}{l}1.4 \\
83\end{array}$ & 14.294 & $\begin{array}{c}20011.3 \\
17\end{array}$ & 25770.024 \\
\hline $\begin{array}{c}160 \\
0\end{array}$ & $\begin{array}{c}2.3 \\
55\end{array}$ & 2.350 & $\begin{array}{l}1.4 \\
52\end{array}$ & $\begin{array}{c}10.8 \\
84\end{array}$ & $\begin{array}{l}1.8 \\
76\end{array}$ & 4.023 & $\begin{array}{c}17.2 \\
58\end{array}$ & $\begin{array}{c}27612.1 \\
06\end{array}$ & $\begin{array}{l}1.5 \\
01\end{array}$ & 14.467 & $\begin{array}{c}23147.6 \\
64\end{array}$ & 29926.872 \\
\hline $\begin{array}{c}180 \\
0\end{array}$ & $\begin{array}{l}2.3 \\
91\end{array}$ & 2.386 & $\begin{array}{l}1.4 \\
68\end{array}$ & $\begin{array}{c}11.0 \\
04\end{array}$ & $\begin{array}{l}1.9 \\
21\end{array}$ & 4.120 & $\begin{array}{c}17.5 \\
10\end{array}$ & $\begin{array}{c}31517.8 \\
88\end{array}$ & $\begin{array}{l}1.5 \\
17\end{array}$ & 14.622 & $\begin{array}{c}26318.7 \\
09\end{array}$ & 34149.759 \\
\hline $\begin{array}{c}200 \\
0\end{array}$ & $\begin{array}{l}2.4 \\
22\end{array}$ & 2.417 & $\begin{array}{l}1.4 \\
82 \\
\end{array}$ & $\begin{array}{c}11.1 \\
09\end{array}$ & $\begin{array}{l}1.9 \\
62 \\
\end{array}$ & 4.208 & $\begin{array}{c}17.7 \\
34 \\
\end{array}$ & $\begin{array}{c}35467.4 \\
99\end{array}$ & $\begin{array}{l}1.5 \\
32 \\
\end{array}$ & 14.766 & $\begin{array}{c}29532.1 \\
63 \\
\end{array}$ & 38420.715 \\
\hline $\begin{array}{c}220 \\
0\end{array}$ & $\begin{array}{l}2.4 \\
48 \\
\end{array}$ & 2.443 & $\begin{array}{l}1.4 \\
95 \\
\end{array}$ & $\begin{array}{c}11.2 \\
06\end{array}$ & $\begin{array}{l}2.0 \\
01\end{array}$ & 4.291 & $\begin{array}{c}17.9 \\
41\end{array}$ & $\begin{array}{c}39469.2 \\
57\end{array}$ & $\begin{array}{l}1.5 \\
46\end{array}$ & 14.901 & $\begin{array}{c}32782.2 \\
43\end{array}$ & 42747.482 \\
\hline
\end{tabular}

Where ( $\left.\mathrm{c}_{\text {aum }}\right)$ specific average heat between $\left(0\right.$ to $\left.\mathrm{t}\left[{ }^{\circ} \mathrm{C}\right]\right)$ at constant humid air pressure in $\left[\mathrm{KJ} / \mathrm{m}^{3}{ }_{\mathrm{N}} \cdot \mathrm{K}\right],\left(\mathrm{c}_{\mathrm{CO} 2}, \mathrm{c}_{\mathrm{N} 2}\right.$ , $\left.\mathrm{c}_{\mathrm{H} 2 \mathrm{O}}\right)$ specific heat at constant pressure of carbon, nitrogen, water vapor dioxide $\left[\mathrm{kJ} / \mathrm{m}^{3}{ }_{\mathrm{N}} . \mathrm{K}\right]$.

$\mathrm{I}_{\mathrm{g}}^{\mathrm{o}}, \mathrm{I}_{\text {aum }}^{\mathrm{o}}$ theoretical enthalpy of combustion gas and humid air at $\left(\mathrm{t}\left[{ }^{\circ} \mathrm{C}\right]\right)\left[\mathrm{kJ} / \mathrm{m}^{3} \mathrm{~N}\right], \mathrm{I}_{\mathrm{g}}$ enthalpy of combustion gas $\left[\mathrm{kJ} / \mathrm{m}^{3} \mathrm{~N}\right]$.

Can finds all specific heat value of fuel gas and gas combustion from special tables for Combustion processes for natural gas fuel $[1,8]$. These mathematical relationships $(1,2,3 \ldots .17)$ relate to the quantities of air and flue gases in the combustion fuel when designing steam generators. These relationships change when the type of fuel used (solid or liquid) changes.

Convert the value Dn from[T/h to $\mathrm{kg} / \mathrm{s}]=\mathrm{Dn} \bullet(1000 / 3600)$

Table 5 


\begin{tabular}{|c|c|c|c|c|c|c|c|}
\hline Dn[T/h] & Dn[kg/s] & Dn[T/h] & Dn[kg/s] & Dn[T/h] & Dn[kg/s] & Dn[T/h] & Dn[kg/s] \\
\hline 10 & 2.778 & 22 & 6.111 & 34 & 9.444 & 46 & 12.778 \\
\hline 12 & 3.333 & 24 & 6.667 & 36 & 10.000 & 48 & 13.333 \\
\hline 14 & 3.889 & 26 & 7.222 & 38 & 10.556 & 50 & 13.889 \\
\hline 16 & 4.444 & 28 & 7.778 & 40 & 11.111 & & \\
\hline 18 & 5.000 & 30 & 8.333 & 42 & 11.667 & & \\
\hline 20 & 5.556 & 32 & 8.889 & 44 & 12.222 & & \\
\hline
\end{tabular}

At pn and th from steam / vapor table $i_{n}=2777.44[\mathrm{KJ} / \mathrm{kg}]$

At $\mathrm{pe}^{-}$and $\mathrm{te}^{-}$from steam $/$water table $\mathrm{ie}^{-}=252.1 \quad[\mathrm{KJ} / \mathrm{kg}]$

Where $\mathrm{ie}^{-}, \mathrm{i}_{\mathrm{n}}$ specific enthalpy of water supply and steam coming out ofthe boiler $[\mathrm{KJ} / \mathrm{kg}][12]$, \{steam tables: are defined as the thermodynamic data that contain the properties of water or steam from charts in [9]\}.

\section{Useful Heat for Every Dn}

Useful heat exchanged in the steam generator between the flue gases and the working agent

$$
Q u=D n \cdot\left(i_{n}-i_{e}^{-}\right)
$$

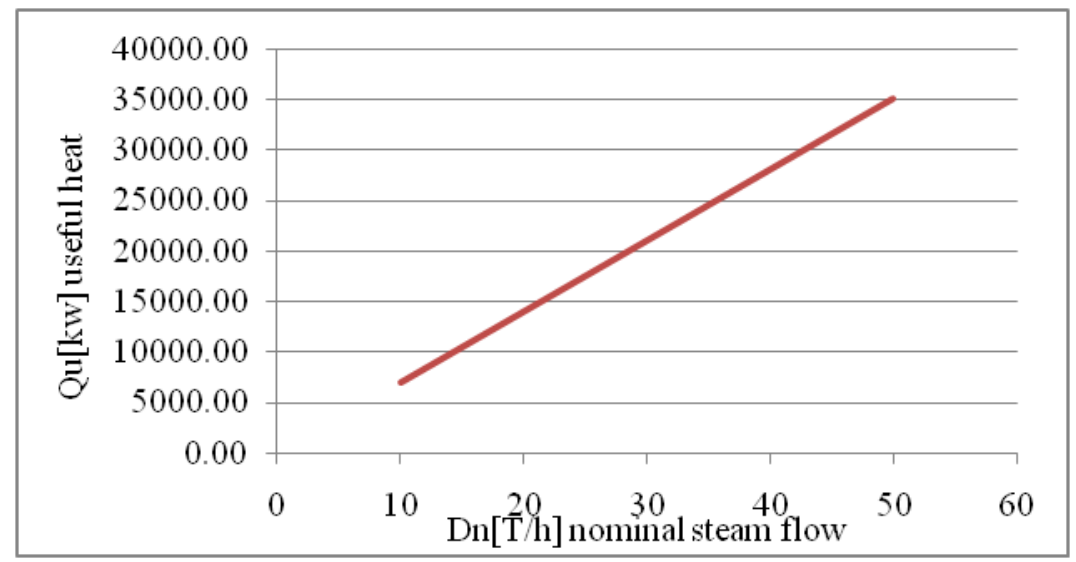

Figure 1: Useful Heat Qu [KW].

We note the linear relationship between the quantity steam produced by the boiler and the amount of useful real heat used for heating (useful heat exchanged in the steam generator between the resulting heat and received).

\section{The $\eta$ of a Generator}

(efficiency output of a generator steam) can find by using value of (qev, qch, qm, qex, qef) equation(19), we note the value of $\mathrm{qm}$ and qch (heat loss for incomplete mechanical and chemical combustion) it depended on characteristics boiler combustion chamber, 1 . For liquid and gaseous fuel with ash $(\mathrm{qm}=0)$ and $(\mathrm{qch}=0.5$ to gas natural and qch $=1.5$ for another type of gas), 2. Sold fuel without ash qm increase between $(0.5-6)$ and (qch $=0)$ depended on type solid fuel. qrf (heat Loss with residues from the furnace), Its value is very small, near zero, for qex (heat loss through walls outside) in Fig .11we notice two curvesdepending on the boiler flow surface (1.Generator without heat exchange surfaces, 2.Generator with heat exchange surfaces), We use the second curve to find values qex for Dn between $(10-50)[\mathrm{t} / \mathrm{h}]$, Value qev calculate from Eq. $(21,22,17,23)$.

$$
\boldsymbol{\eta}=100-(q e v+q c h+q m+q e x+q e f)
$$


for gas natural $(\mathrm{qm}=\mathrm{qrf}=0)$, $\mathrm{qch}=0.5 \%$ constant.

$q e v[\%]=\frac{\left(\frac{Q e v}{Q i^{u m}}\right)}{100}$

$\lambda=1.1$ from Table 4,Between $\left(\operatorname{Ig}\left(200^{\circ} \mathrm{C}\right), \operatorname{Ig}\left(400^{\circ} \mathrm{C}\right)\right)$ calculating $\operatorname{Ig}$ at tev $=220\left[{ }^{\circ} \mathrm{C}\right]$.

Between $\left(\mathrm{I}^{\circ}\right.$ aum $\left(0^{\circ} \mathrm{C}\right), \mathrm{I}^{\circ}$ aum $\left.\left(100^{\circ} \mathrm{C}\right)\right)$ calculating $\mathrm{I}^{\circ}$ aum at to $=25\left[{ }^{\circ} \mathrm{C}\right]$.

$i c o=C c^{u m} \cdot t o$

$Q e v=\left\{I g[\lambda e v, t e v]-\lambda e v \cdot \mathrm{I}_{\text {aum }}[t o]\right\}-i c o$

To find qev (heat loss with exhaust gas) use Eq.(3, 21,22,23) where (Qev) the heat loss $\left[\mathrm{kJ} / \mathrm{m}^{3} \mathrm{~N}\right]$, (ico) specific enthalpy of fuel at ambient temperature[ $\mathrm{kJ} / \mathrm{m}^{3}{ }_{\mathrm{N}}$, qex from fig. 11 find every $\eta(\mathrm{Dn})[\%]$ see table 5.

\begin{tabular}{|c|c|c|c|c|c|}
\hline $\operatorname{Dn}[\mathrm{T} / \mathrm{h}]$ & qex & {$[\%] \eta$} & $\mathrm{Dn}[\mathrm{T} / \mathrm{h}]$ & qex & {$[\%] \eta$} \\
\hline 10 & 1.7 & 88.741741 & 30 & 1.22 & 89.221741 \\
\hline 12 & 1.6 & 88.841741 & 32 & 1.2 & 89.241741 \\
\hline 14 & 1.5 & 88.941741 & 34 & 1.2 & 89.241741 \\
\hline 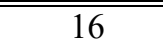 & 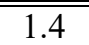 & 89.041741 & $\overline{c 36}$ & "1.1 & 89.341741 \\
\hline 18 & 1.3 & 89.141741 & 38 & 1 & 89.441741 \\
\hline 20 & 1.28 & 89.161741 & 40 & 1 & 89.441741 \\
\hline 22 & 1.27 & 89.171741 & 42 & 0.98 & 89.461741 \\
\hline 24 & 1.26 & 89.181741 & 44 & 0.96 & 89.481741 \\
\hline 26 & 1.25 & 89.191741 & 46 & 0.94 & 89.501741 \\
\hline 28 & 1.24 & 89.201741 & 48 & 0.92 & 89.521741 \\
\hline \multicolumn{6}{|r|}{89.541741} \\
\hline
\end{tabular}

\section{Nominal Flow of Natural Gas}

We find nominal flow of natural gas $\mathrm{Bg}\left[\mathrm{m}^{3} \mathrm{~N} / \mathrm{s}\right]$ by using Eq.(18, 19, 2, 3, 24) see figure2.

$$
B g(D n)=\frac{Q u(D n)}{0.01 \cdot \eta(D n) \cdot Q i_{u m}}
$$

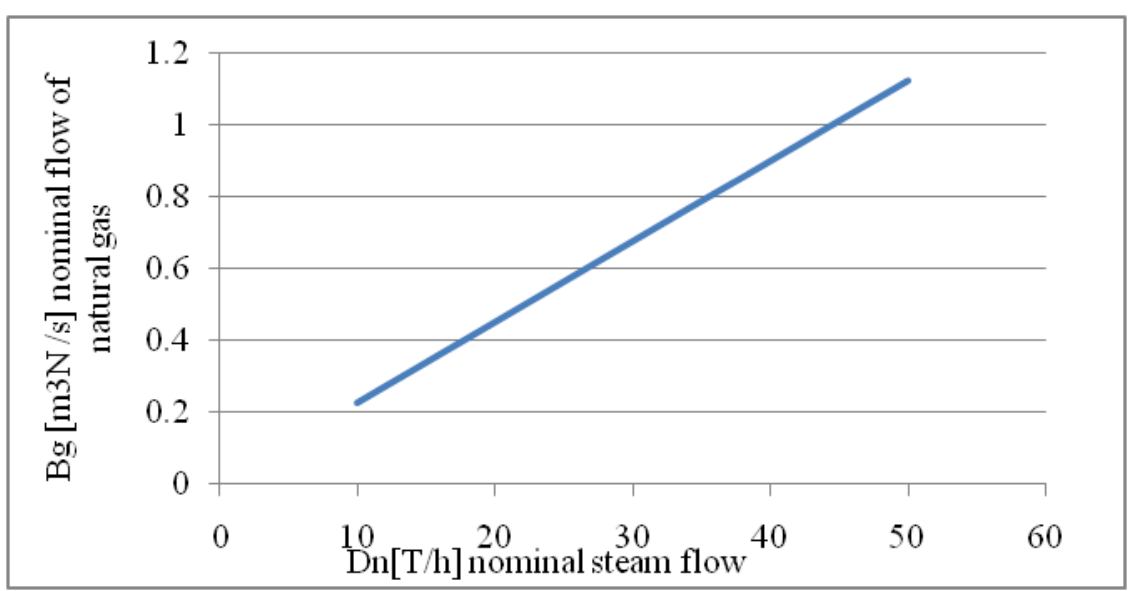

Figure 2: Nominal Flow of Natural Gas Bg $\left[\mathrm{m}^{3} \mathrm{~N} / \mathrm{s}\right]$.

In this graph, it shows the true relationship between the increase in the amount of gas used for the combustion and 
heating process and the steam production.

\section{Air Flow Circulated in the Burner}

By use Eq. $(4,5,6,24,25)$, coefficient for exhaust air $\lambda$ and temperature of the preheated air Tp̈ in kelvin.

$$
D a=\lambda \cdot V_{a u m}^{0} \cdot B g(D n) \cdot\left(\frac{T \not p}{273}\right)
$$

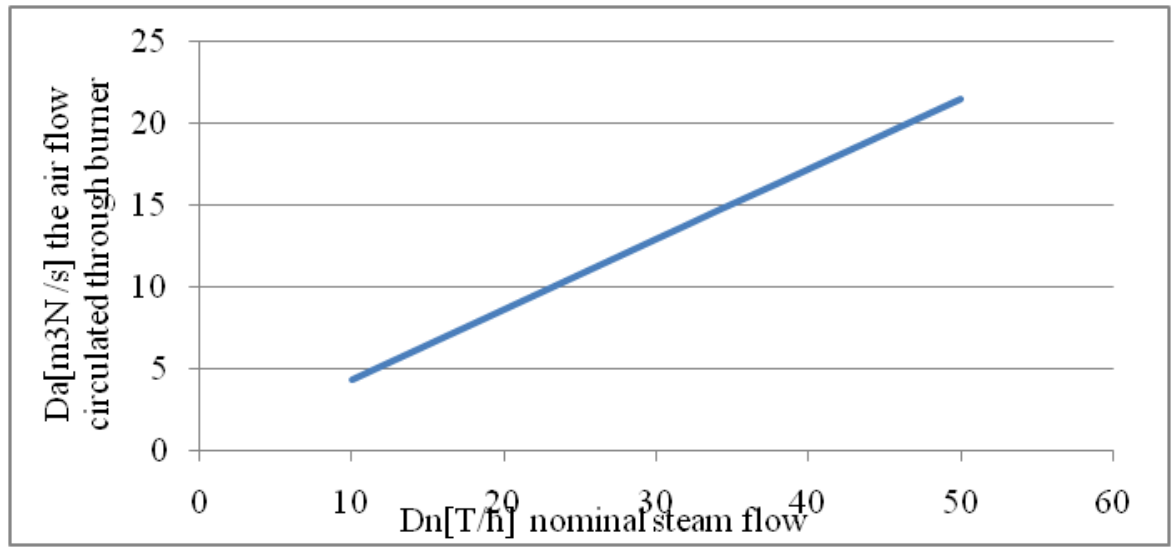

Figure 3: Air Flow Circulated in the Burner $\mathrm{Da}\left[\mathrm{m}^{3} \mathrm{~N} / \mathrm{s}\right]$.

To increase the amount of heat produced during the combustion process, the amount of air entering the burner must be increased, along with an increase in the amount of gas for the occurrence of perfect combustion.

\section{The Air Flow Nozzle}

Required average air flow rate wa $=20$ [m/s][14],It is constant value and use Eq. $(25,26,27)$ we find cylindrical cross section and diameter see figure. 14 .

$$
S a=\frac{D a}{w a}
$$

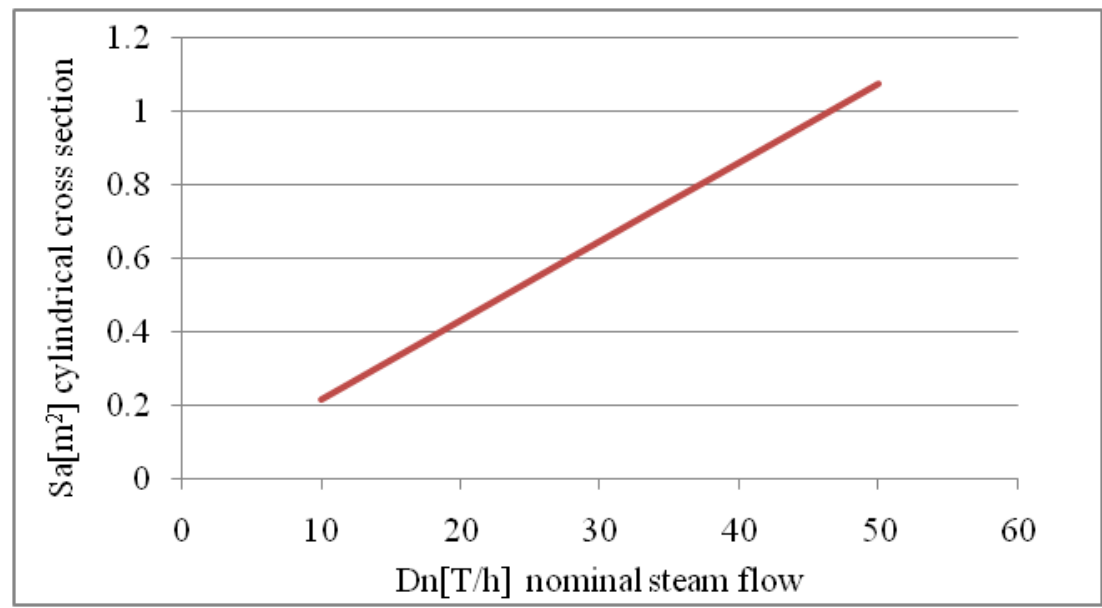

Figure 4: Cylindrical Cross Section $\mathrm{Sa}\left[\mathrm{m}^{2}\right]$.

The area of the air transporter tube (the air flow nozzle) increases with the increase in the amount of steam produced to maintaining the stability of the burner flame and the stable distribution of heat. 
$d a[m]=\frac{\sqrt{4}}{\pi} * S a$

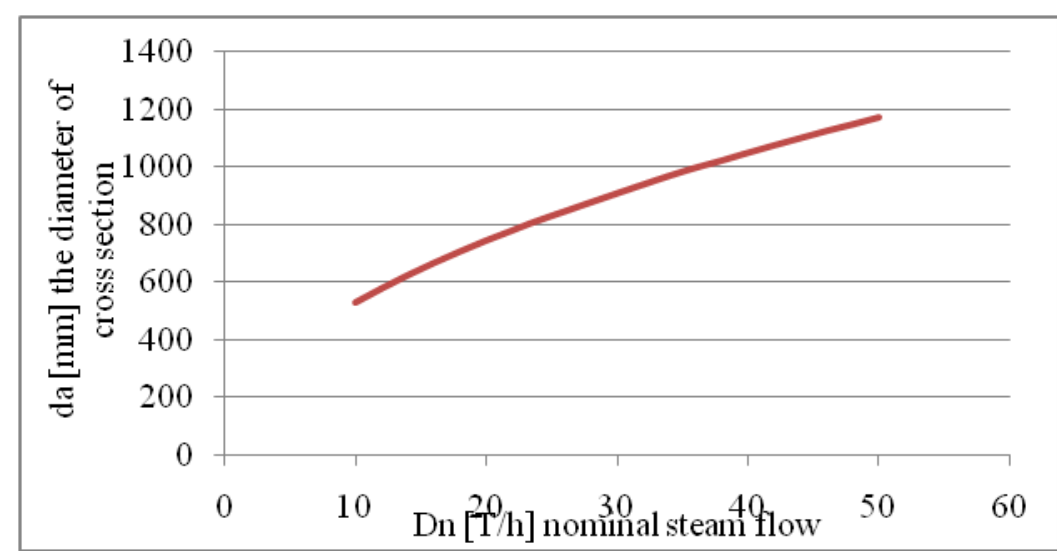

Figure 5: Diameter of Cross-Section da [m].The Diameter Increases with Increasing Area.

\section{The Outer Diameter of the Swirl Register}

By use Eq. $(26,27,28,29)$ and you should find $\gamma$ (considered pallets by angle at the center) it depend on number of the blades (nb) and the tilt angle of the blades $(\theta)$.we have two value of $(\gamma, \mathrm{D})$ if $\theta=30^{\circ} \mathrm{nb}=18$ and $\theta=45^{\circ}, \mathrm{nb}=24$ see figure 15

$$
\begin{aligned}
& \gamma=\frac{360}{n b} \\
& D=d a \frac{\cos \theta}{\cos (\theta+0,7 \gamma)}
\end{aligned}
$$

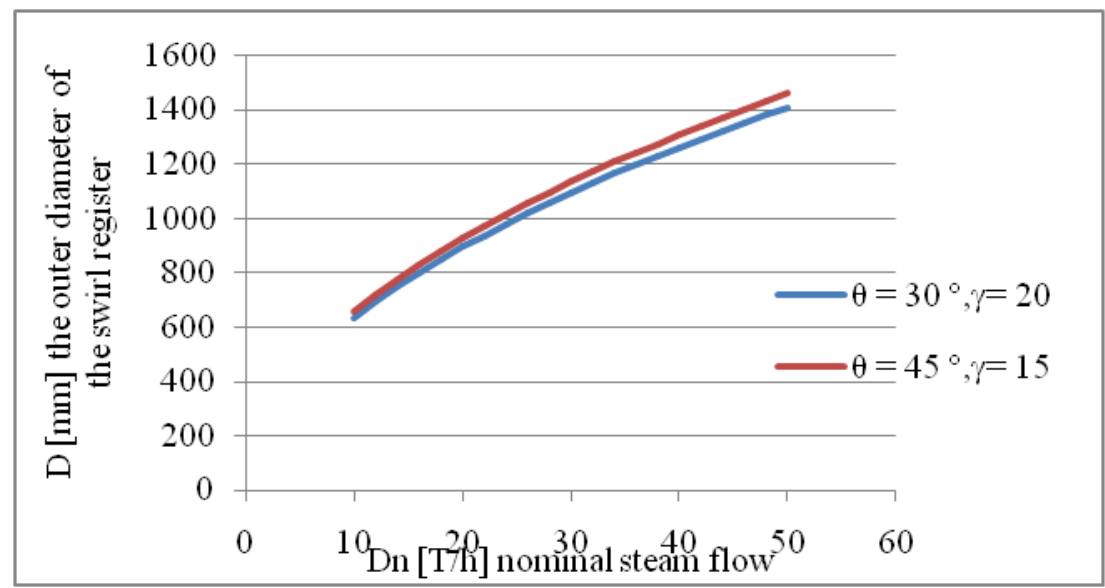

Figure 6: Outer Diameter of the Swirl Register D [mm].

The increase in the diameter of the circle in relation to the tilt angle of the blades o in order to avoid occurrence pressure drop in flow gas inlet to the burner .

The Thickness between the Blades

$$
\varepsilon=d a * \sin \frac{180}{n b} \cdot \sin \left(\theta+\frac{180}{n b}\right)-\delta
$$

Where $\delta=3 \mathrm{~mm}$ Correction coefficient 


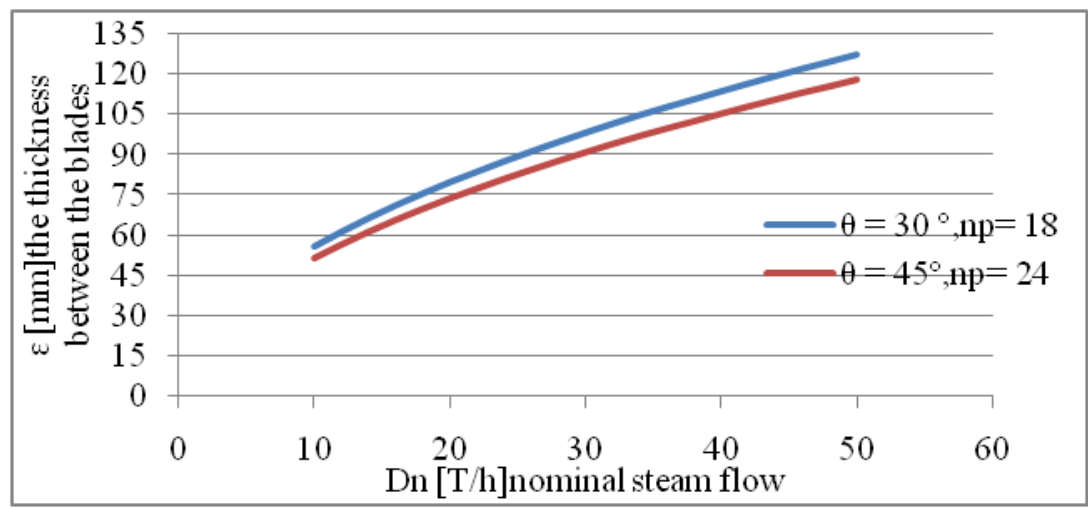

Figure 7: The Thickness between the Blades $\varepsilon[\mathrm{mm}]$.

\section{Working Length of the Blade}

$$
L=1.6 \cdot \frac{d^{2} a}{n p * \varepsilon} \cdot \cos \theta
$$

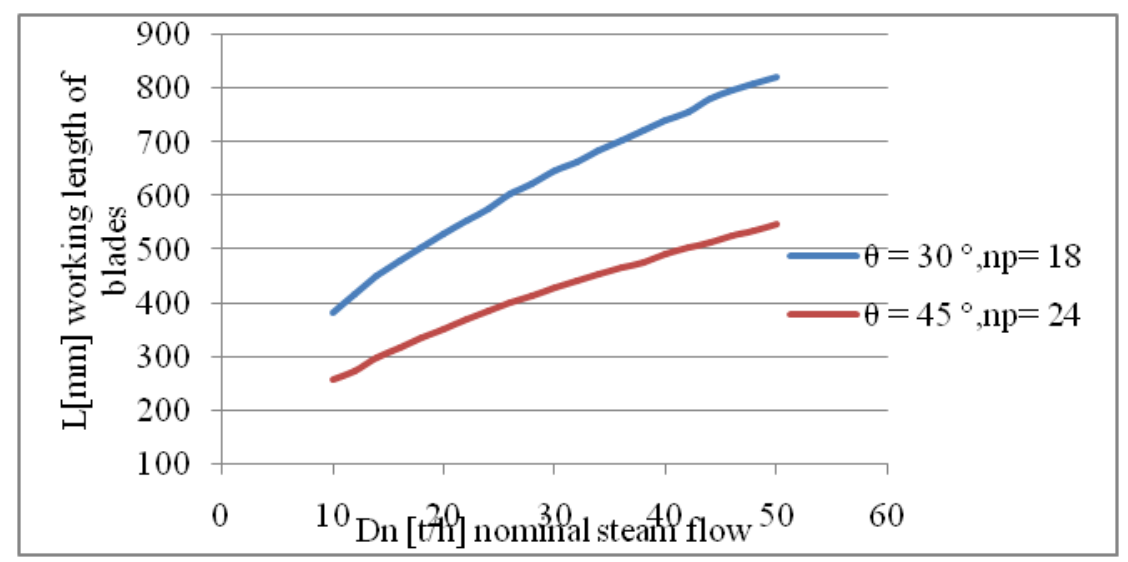

Figure 8: Working Length of the Blade $L$ [mm].

The Length of the Housing Air

$$
L c=L \cdot l t
$$

Where $1 \mathrm{t}=0.25[\mathrm{~mm}]$ constant (the length of the burning neck).

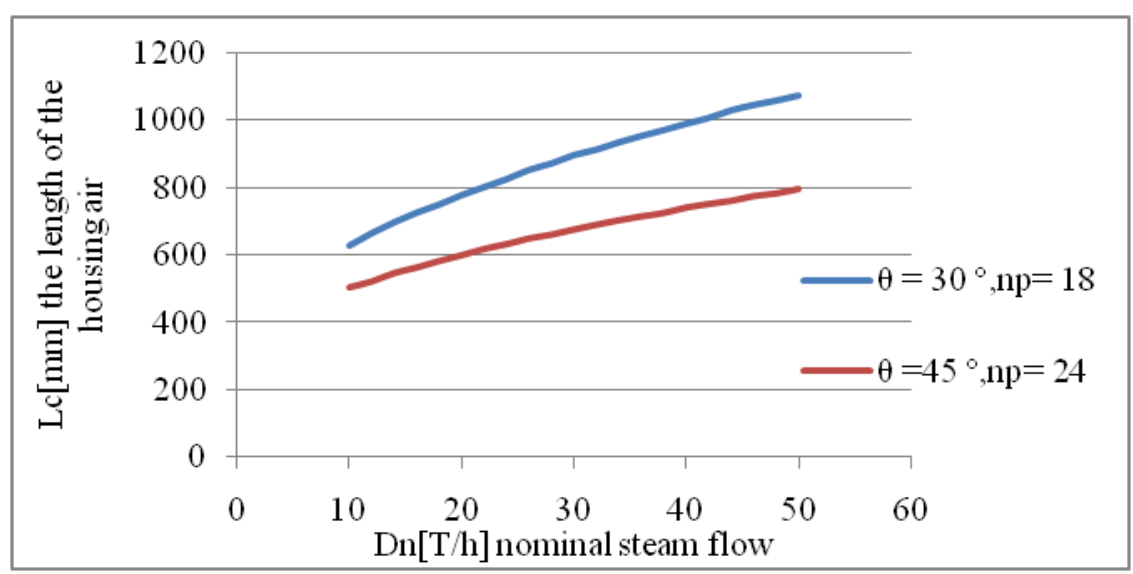

Figure 9: Working Length of the Blade Lc [mm]. 
The Maximum Diameter of the Housing Air

$$
D e=\frac{0,785 * d a^{2}}{L c}+D
$$

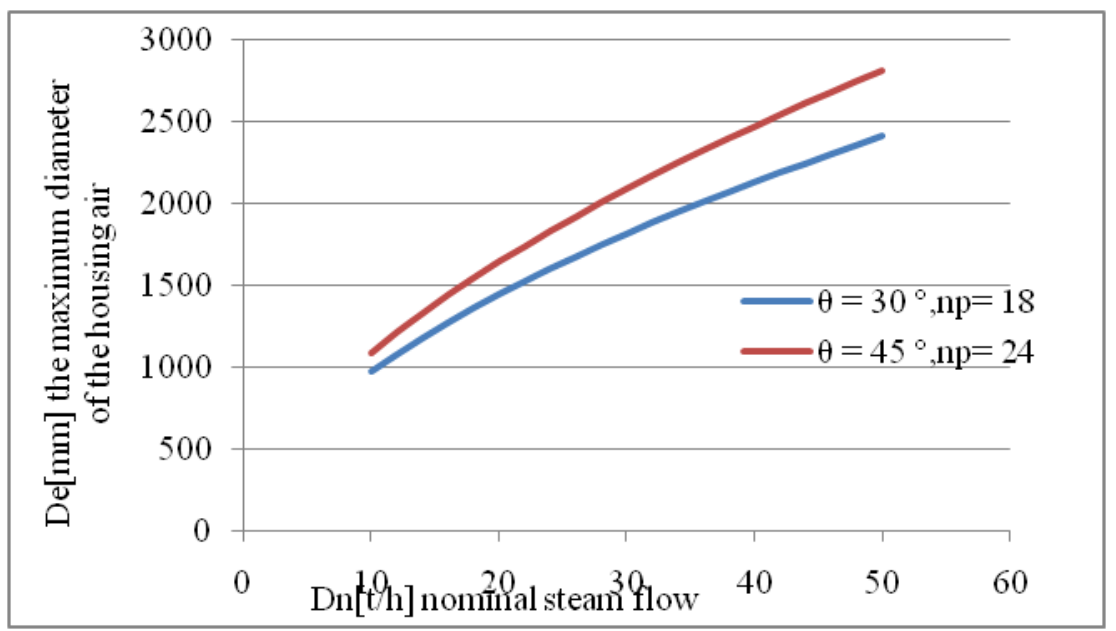

Figure 10: Maximum Diameter of the Housing De [mm].

We learned how to find the values for $\left\{\mathrm{Qu}[\mathrm{KW}]\right.$, The $\eta$ of a generator, $\mathrm{Bg}\left[\mathrm{m}^{3} \mathrm{~N} / \mathrm{s}\right], \mathrm{Da}\left[\mathrm{m}^{3} \mathrm{~N} / \mathrm{s}\right]$ and da $\left.[\mathrm{mm}]\right\}$.We can use these values to choose the appropriate type and size of the burner for the boiler, and this requires time and effort and affects the speed of work and production. So depending on the amount of steam to be produced (boiler capacity Dn[T/h]) and without resorting to using a mathematical equation, we have graphs that can extract all the values needed for the design directly [3].

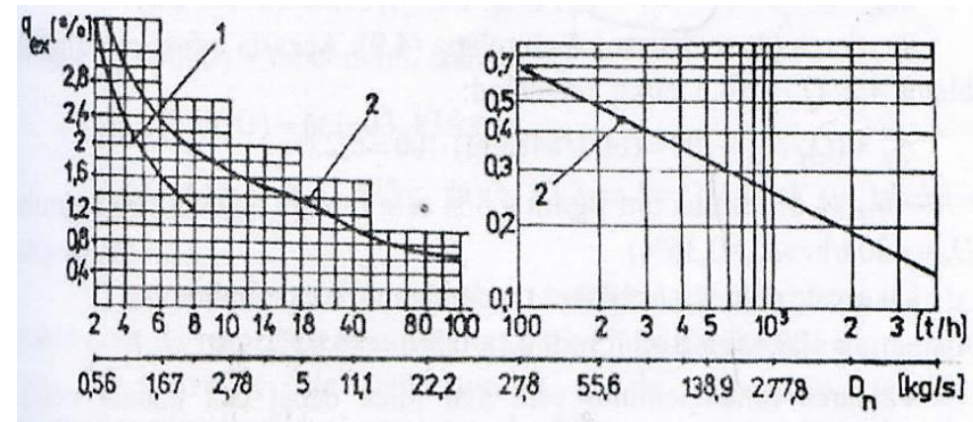

Figure 11: Determination of the Percentage Heat Loss Outside [1].

1. Generator without Heat Exchange Surfaces, 2 .Generator with Heat Exchange Surfaces.

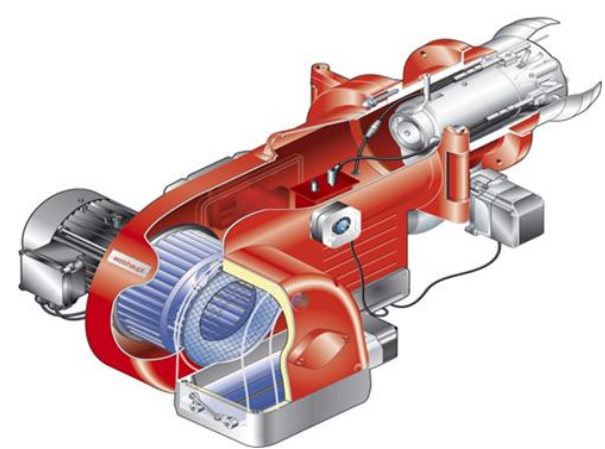

Figure 12: High Pressure Gas Burner. [3]. 

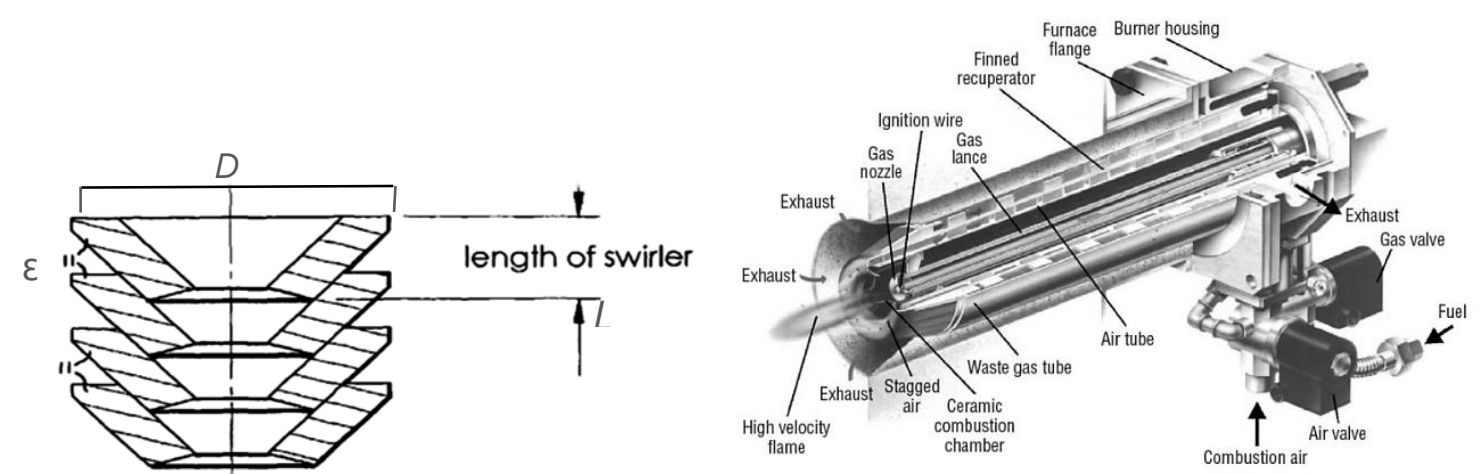

Figure 14: Cross Section of a Blades[15] Figure 13: An Internal Section of a Kind Gas Burner[14].

\section{CONCLUSIONS}

For a certain size of fuel gas, they should have a high consumption rate. High consumption means less manufacturing costs and allows the heat to be produced which is usually most efficient, So to maintain the ratio of air/gas mixing inside the burner[13], the transmission piping diameters should not be kept too large for the flame spread speed to exceed the speed of the mixing process, and the flowing mixture speed should not be too high so that the complete combustion process does not take place and maintain the speed of entry of air and gas flow The flame of the burner must be uniform in height and uniformly distributed inside the furnace, Control the internal gas and air velocity to make the mixing process homogeneous to avoid an explosion.[6,7]

\section{ACKNOWLEDGMENTS}

I would like to thank my supervisor \{Prof. Dr. Ing. Ionel Gh. Pîșă \}. Head of the Thermal Equipment Department of the Polytechnic University of Bucharest / Faculty of Mechanical and Mechatronics Engineering for continuous follow-up, scientific guidance and assistance in giving accurate feedback.

\section{REFERENCES}

1. Pîşăa, I. (2013). Generatoare de abur. Politehnica Press.

2. Platvoet, E., \& Baukal, C. (2013). Process burners 101. Chemical Engineering Progress, 109(8), 35-39.

3. C.I.B. UNIGAS S.p.A Industrial burners Liquid catalog and price list. URL: http://www.cibunigas.com/ru/product-catalogs [in Russian].

4. Zimont, V., Moreu, V., Battaglia, V., \&Modi, R. (2011). RANS and LES Modeling of the GE10 Burner. Energy and Power engineering, 3(05), 607.

5. Eiseman, J. H., Weaver, E. R., \& Smith, F. A. (1932). Method for Determining the Most Favorable Design of Gas Burners. Bureau of Standards Journal of Research, 8.

6. Gattei, L. (2009). A study on the fluid dynamics of domestic Gas burners (Doctoral dissertation, alma).

7. Berry, W. M., Brumbaugh, I. V., Moulton, G. F., \& Shawn, G. B. (1921). Design of atmospheric gas burners (No. 193-202). US Government Printing Office.

8. Riello S.p.A., “Technical Handbook of the RG1 Burner," Via degliAlpini, 1-37045 Legnago (VR) Italia, 2001.

9. Meyers, C. H., Cragoe, C. S., \& Mueller, E. F. (1947). Table and Mollierchart of the thermodynamic properties of 1, 3 - 
butadiene. J ResNatlBur Stand, 39, 507-507.

10. Reitz, R. D., Ogawa, H., Payri, R., Fansler, T., Kokjohn, S., Moriyoshi, Y., ... \&Boulouchos, K. (2020). IJER editorial: The future of the internal combustion engine.

11. Noor, M. M., Wandel, A. P., \&Yusaf, T. (2013). Design and development of mild combustion burner. Journal of Mechanical Engineering and Sciences, 5, 662.

12. Denev, J. A., Dinkov, I., \&Bockhorn, H. (2017). Burner design for an industrial furnace for thermal postcombustion. EnergyProcedia, 120, 484-491.

13. Gattei, L. (2009). A study on the fluid dynamics of domestic Gas burners (Doctoral dissertation, alma).

14. Good, M., \& Sense, E. (2019). High-Efficiency Gas Burners.

15. Mao, C. P., \& Short, J. E. (2014). U.S. Patent No. 8,800,146. Washington, DC: U.S. Patent and Trademark Office. 
